\title{
Dielectric barrier discharges and ozone synthesis
}

\author{
Gerhard J. Pietsch ${ }^{\mathrm{a}}$ and Valentin I. Gibalov ${ }^{\mathrm{b}}$ \\ aAachen University of Technology, Schinkelstr. 2, D-52056 Aachen, Germany \\ ${ }^{b}$ Moscow State University, Chemical Department, Moscow, 119899, Russia \\ Abstract: The discharge structure, development and the transferred charge of \\ dielectric barrier discharges (DBD) in arrangements with a gas gap (volume discharge, \\ VD) and in such with pure surface discharges (SD) are compared. On the basis of the \\ properties of DBD some parameters influencing efficiency and ozone production like \\ field strength distribution and energy density are discussed. An explanation for \\ observed unexpected low ozone yields in air-fed SD generators is proposed.
}

\section{INTRODUCTION}

Applying ac voltage to an electrode system with one or both electrodes covered by a dielectric layer, a dielectric barrier discharge (DBD) appears in the gas gap. Such non-thermal discharges are suitable for a wide range of applications, e. g. ozone generation, surface treatment and modification of plastic foils, textiles and even metals, pollution control, sterilisation, ultraviolet and vacuum ultraviolet light sources for pumping of laser, ac plasma displays and others.

The basic designs of plasma chemical reactors using DBD are given in Fig. 1. While the DBD in Fig. $1 \mathrm{a}$ is mainly determined by discharge columns in the gas gap and surface discharges on the dielectric, the arrangement with extended surface electrodes (Fig. $1 \mathrm{~b}$ ) leads only to surface discharges along the dielectric surface. The packed bed reactor with dielectric pellets (Fig. $1 \mathrm{c}$ ) includes both types of discharges.

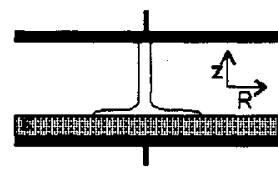

a)

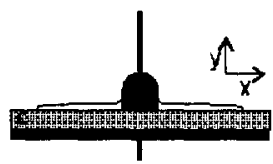

b)

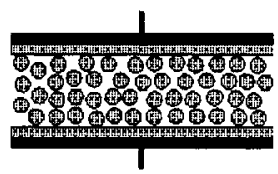

c)

Fig. 1 Cross section of different types of DBD arrangements a) with volume,

b) with surface discharges and c) packed bed reactor

In this contribution some properties of DBD in the volume discharge (VD) and in the surface discharge (SD) devices at atmospheric pressure are considered. Some aspects of ozone synthesis in these arrangements are discussed.

\section{PROPERTIES OF DBD}

\section{Discharge structure and transferred charge}

Due to the presence of a dielectric in the discharge arrangements, the DBD at atmospheric pressure consists in the VD device (Fig. 1 a) in general of tiny microdischarges, which are randomly distributed within the discharge gap in time and space. In SD devices (Fig. 1 b) the DBD propagates stepwise perpendicular to the surface electrode so that distinct current pulses can be detected. The dielectric distributes the microdischarges over the entire electrode area and limits the amount of charge and energy of each discharge 
pulse. The electrical energy coupled into the gas is mainly used to produce energetic electrons, while the gas remains at nearly ambient temperature.

The discharge patterns on the dielectric for VD as well as SD depend on polarity. In both arrangements the accumulation of positive ions on the dielectric surface leads to discharge channels covering the dielectric, the accumulation of electrons to a homogeneous discharge structure.

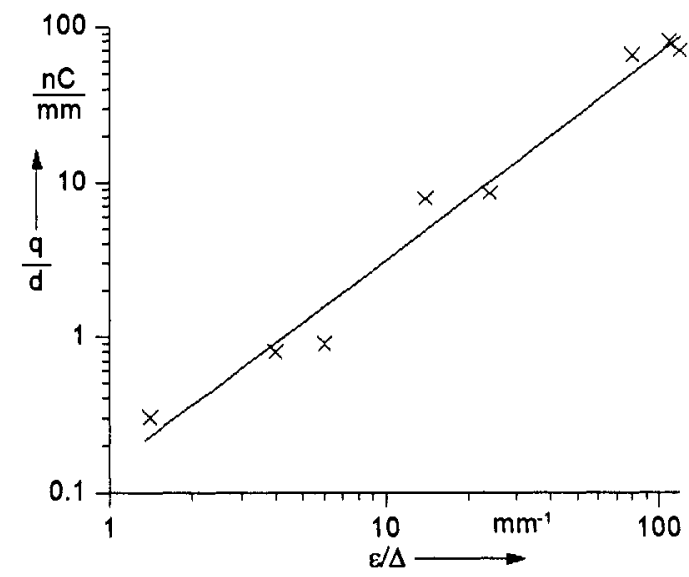

Fig. 2 Measured values of the related transferred charge $q$ of a microdischarge versus specific capacity of the dielectric in oxygen of 1 bar ( $d$ - width of gas gap, $\varepsilon$ and $\Delta$ - relative permittivity and thickness of the dielectric, respectively).

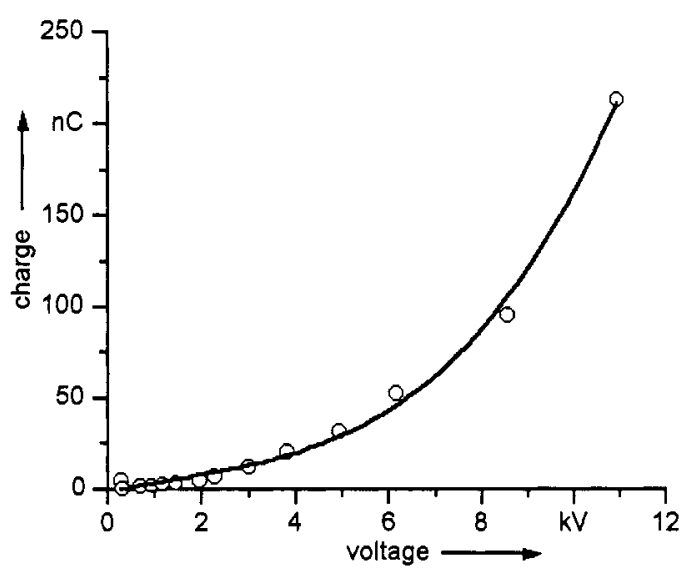

Fig. 3 Measured values of transferred charge from a period of applied voltage of a SD arrangement (electrode length $6 \mathrm{~cm}$, oxygen of 1 bar) versus voltage amplitude.

In electrochemical processes chemical transformations are in general proportional to the amount of charge flowing through the reactor. In DBD reactors the electron current is most important for the number of generated excited particles and radicals. In VD arrangements the average current is defined by the number of microdischarges each discharging a small area on the dielectric. Rising the voltage amplitude this number rises linearly and by this the mean current and the transferred charge. The amount of charge transferred within a single microdischarge depends apart from the process gas on the capacity of the dielectric layer, and in VD devices on the width of the gas gap (Fig. 2).

In SD arrangements the discharged area (capacity) is proportional to the voltage amplitude. As the charge is the product of capacity and voltage the transferred charge of a period of voltage is proportional to the square of the applied voltage (Fig. 3).

\section{Discharge development}

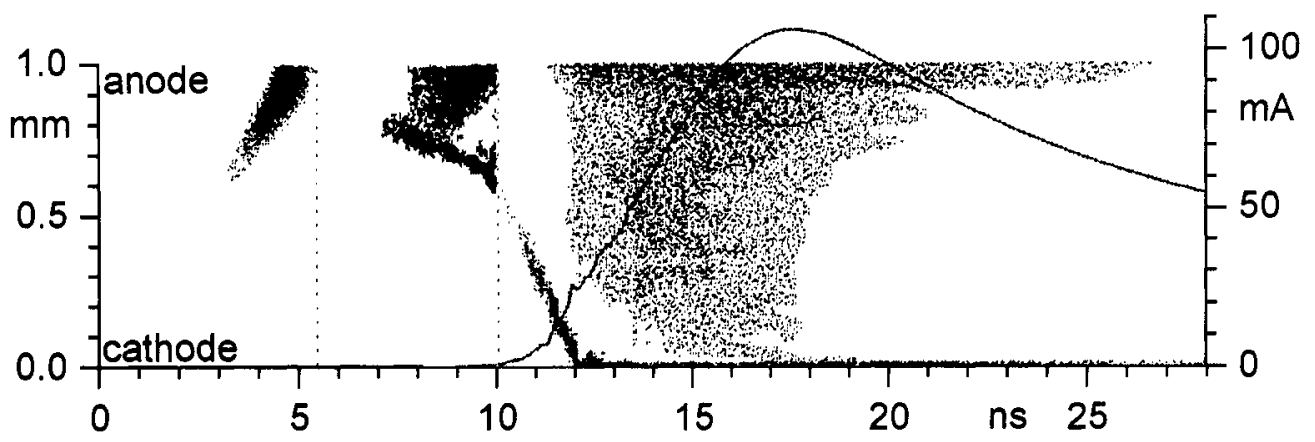

Fig. 4 Calculated streak photograph of a microdischarge in air at atmospheric pressure (dielectric anode) together with the corresponding current slope (at the dotted lines the sensitivity is changed)

The temporal development of microdischarges (Fig. 4) and the discharge pulses in SD devices can be divided into several phases. The initial phase of the VD is determined by a homogeneous initial field strength 
distribution, while in SD devices it is strongly non-linear (Fig. 5). Within the intermediate phase of VD, especially in electronegative gases, a self amplifying process starts leading to a cathode directed streamer with mean electron energies around $10 \mathrm{eV}$ and a well developed cathode layer (Fig. 6). Its area determines the current development. In SD no distinct cathode layer has been found (ref. 1).
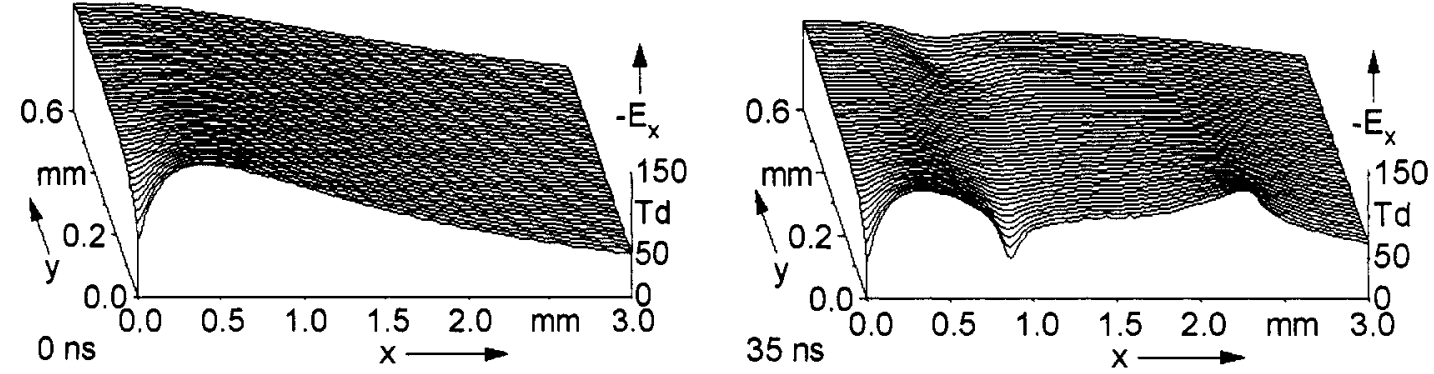

Fig. 5 Distribution of the field strength component parallel to the dielectric surface in a SD arrangement (Fig. $1 \mathrm{~b}$ ) at negative polarity of the surface electrode, initial distribution $(0 \mathrm{~ns})$ and during the final phase of the discharge pulse ( $35 \mathrm{~ns})$.

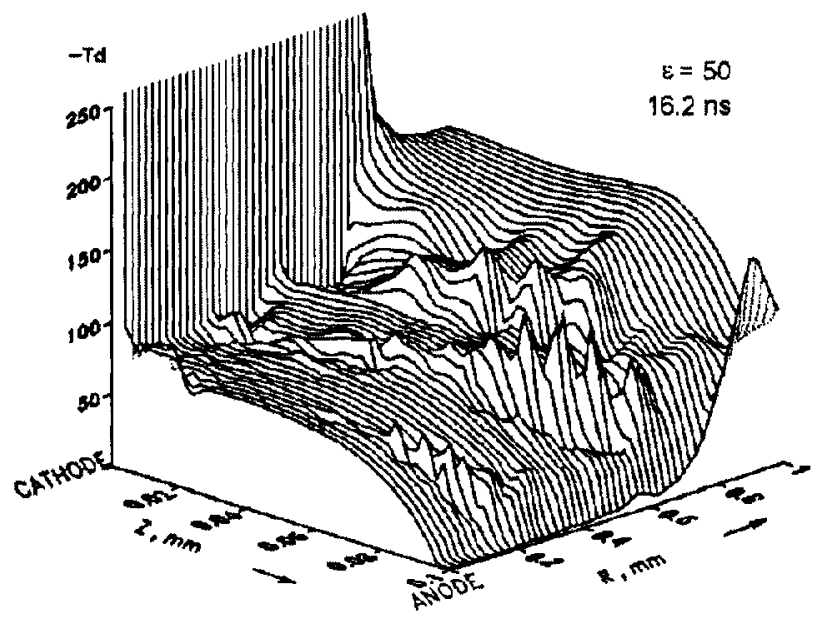

Fig. 6 Calculated axial field strength distribution of a microdischarge during current peak (dielectric anode).
During the next phase the main part of the charge is transferred. The conductivity grows. The mean electron energy is a few $\mathrm{eV}$. Depending on the arrangement under consideration (Fig. 1 $\mathrm{a}$ or $\mathrm{b})$ the field strength within the conducting region drops and grows, respectively, to a value where the effective ionisation coefficient is close to zero (Fig. 5 and 6). In SD devices a field strength peak is moving along the surface. The main part of radicals and excited particles is created within this phase of the discharge. The growing amount of charge carriers on the dielectric extinguishes the discharge and it decays finally.

In general the duration of the main physical processes are rather short in comparison with chemical reactions. Therefore physics and chemistry in plasma chemical reactors can often be dealt with separately.

\section{ASPECTS OF OZONE SYNTHESIS}

\section{Improvement of efficiency}

The main goals of research in the field of ozone generation are improvement of the efficiency, the increase of production per unit volume of the reactor and for some applications the achievement of high ozone concentrations.

As the ozone generation starts with electron collision processes leading to free oxygen atoms, optimal field strength conditions have to be achieved. This optimum has been found out experimentally (Fig. 7). It is nearby the breakdown value of microdischarges in normal, commercial ozone generators. 


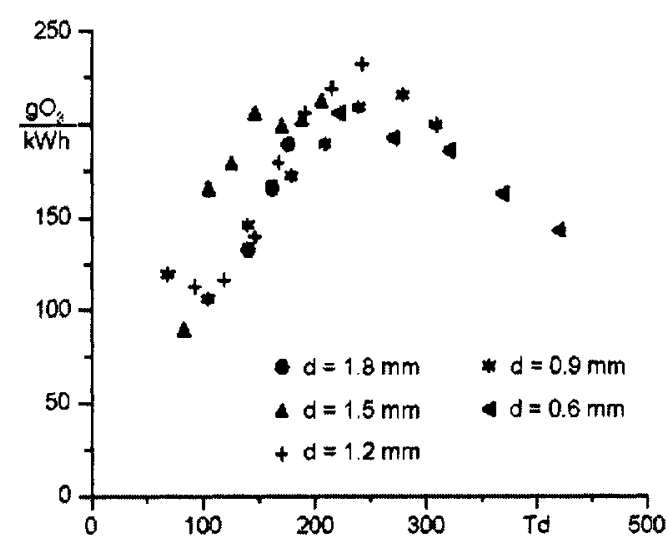

Fig. 7 Measured ozone yield versus reduced field strength of non self-sustaining discharges in oxygen at $5 \mathrm{kPa}$ (for several gas gap widths $d$, ref. 2).

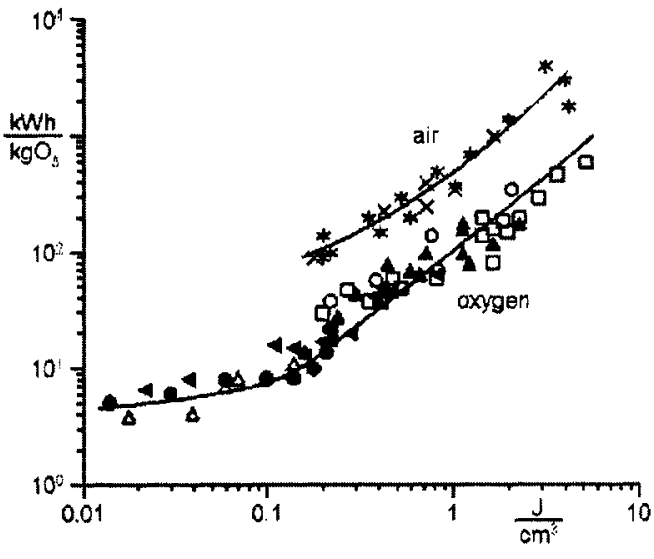

Fig. 8 Energy consumption of ozone yield depending on energy density in the discharge (for different gas gaps and pressures of a VD device, ref. 2).

However, the initial field strength is roughly halved during the development of the discharge (Fig. 6) connected with an increase of loss processes in the discharge (Fig. 9). In a limited range it is possible to tailor the VD by reducing the discharge gap and the dielectric constant or enlarging the thickness of the dielectric layer. The result of these measures are a reduction of the discharged area and of the specific capacity, respectively, leading to low developed, faint discharge channels with corresponding higher field strengths.

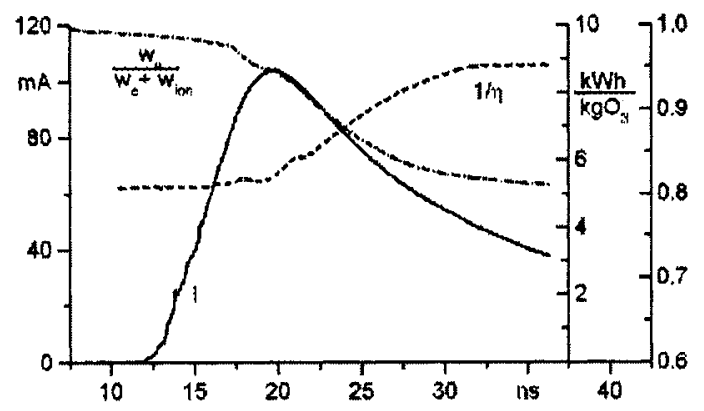

Fig. 9 Calculated values of the mean electron energy portion $w_{e} /\left(w_{e}+w_{i o n}\right)$, energy consumption of ozone yield $l / \eta$ and the current slope $I$ of a microdischarge in air at atmospheric pressure during the variation with time, initial field strength $185 \mathrm{Td}$.
For SD arrangements the situation is a bit different. Although the elementary physical processes are the same, there exists no distinct gap width. The discharge is developing into a decreasing field strength. From modelling results it follows that the field strength in the outer regions of the discharge will rise up to the value where the effective ionisation coefficient is about zero (Fig. 5). That is why low field strengths are predominant and it is to be expected that ozone synthesis efficiency with SD will not exceed that of optimized VD devices.

\section{Production rate}

In general the ozone yield is determined by the energy density within the discharge. There exists a wide range of proportionality of energy density and oxygen or ozone concentration. In Fig. 10 this behaviour is shown for VD (Fig. $1 \mathrm{a}$ ) and SD arrangements (Fig. 1 b). The deviation of this proportionality in the cathode layer region of the microdischarge results from the high energy density of ions in the cathode layer which are not able to dissociate molecular oxygen effectively.

An increase of ozone production will be achieved by an enlargement of the energy density. This is possible by increasing the number of charge pulses (frequency, voltage amplitude) or the amount of charge of the single pulses (Fig. 2). However, there exist limitations. With growing energy density of the discharge the reduced field strength in the discharge channels will lead to enlarged ion losses on one hand and on the other hand it will rise the temperature of the process gas. The chemical reaction which yields ozone strongly depends on 
temperature. Energy densities above $100 \mathrm{~mJ} / \mathrm{cm}^{3}$ reduce efficiency as well as ozone production dramatically (Fig. 8)
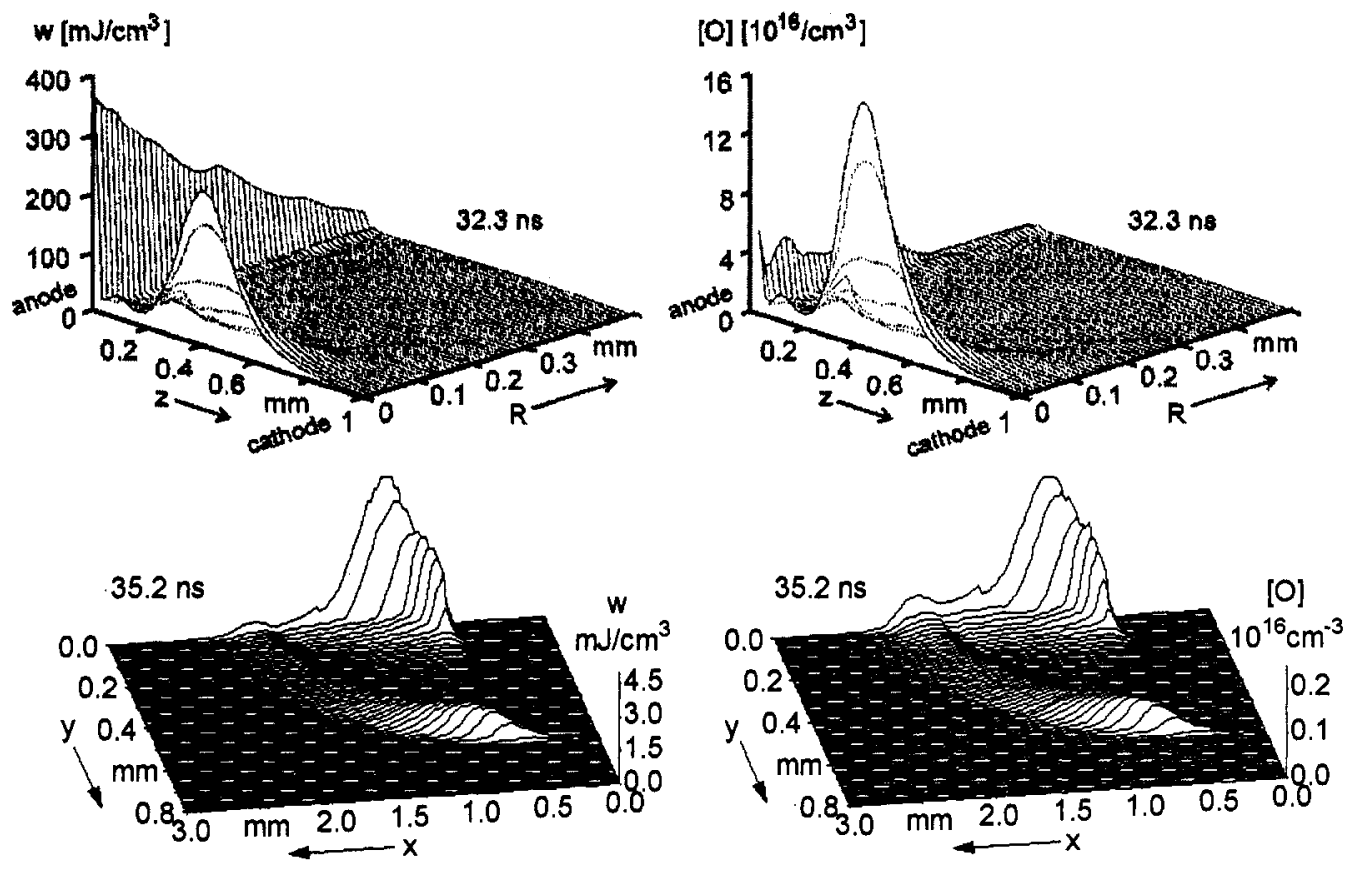

Fig. 10 Calculated distributions of energy density and oxygen concentration in air at atmospheric pressure in a VD (top) and in a SD arrangement (bottom).

High efficiency, production density and concentration are partly independent from one another. Apart from field strength and energy density distributions the chemical reaction scheme as well as the overall reactor behaviour have to be considered, especially the interaction of the discharge pulses with the process gas.

\section{Ozone generation with VD and SD arrangements}

It is well known that ozone synthesis from oxygen results in higher ozone concentrations and efficiencies than that from air. In air especially at high energy densities in the reaction volume, even a poisoning of the ozone synthesis may occur (ref. 3). This can be explained by ozone destruction processes via catalytic reaction chains with low nitrogen oxides.

In commercial, well optimized VD ozone generators the ozone yield from oxygen is about a factor of two to three higher than from air. In commercial SD ozone generators, however, the difference in the yield is much more pronounced and exceeds in most cases a factor of four (Fig. 11). That is why SD generators are in general fed with oxygen. In this case a frequency rise will enlarge the production rate as long as cooling prevents a drastic temperature rise and by this a thermal decomposition of ozone.

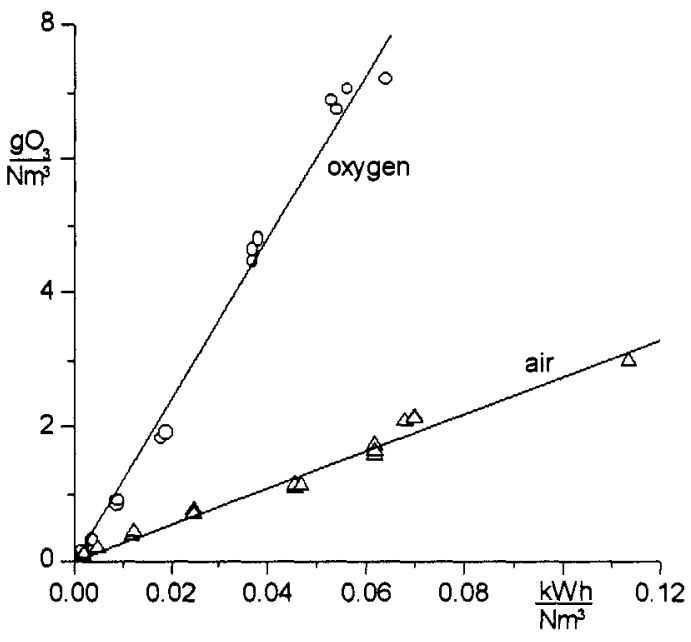

Fig. 11 Measured ozone concentrations versus energy density of a SD ozone generator model. 
The difference in the ozone yield from VD and SD generators is up to now not understood. An explanation may be the following. In general VD generators work with frequencies of the applied voltage of less than 1 $\mathrm{kHz}, \mathrm{SD}$ generators with frequencies of about $10 \mathrm{kHz}$. At these frequencies the slowest reaction in the reaction chain to high nitrogen oxides has a time constant which is long in comparison with the duration of a half period. The equilibrium between lower and higher nitrogen oxides can therefore be shifted towards the lower nitrogen oxides with an increased decomposition of ozone by catalytic cycles.

\section{CONCLUSIONS}

Plasma chemical reactors with dielectric barrier discharges (DBD) are simple in device and operation. The $\mathrm{DBD}$ is an efficient source of high energetic electrons which are able to initiate plasma chemical reactions. It can easily be sustained even at atmospheric pressure. The DBD consists of numerous self pulsing microdischarges in a gas gap (volume discharge, VD) with corresponding surface discharges on the dielectric and of a stepwise growth of discharge structures on dielectric surfaces, when pure surface discharge (SD) arrangements are considered. The time constants of these processes are in the nanosecond range. The field strength distribution in the discharge channels changes with time. Its initial shape depends on the arrangement under consideration.

In order to get high efficiencies of the ozone synthesis, from a physical point of view the field strength within the discharge structures should be near the optimal value which has been found experimentally. The field strength distribution during the main charge transfer can be tailored to a certain extend by an appropriate choose of the gap width and the specific capacity of the dielectric. These parameters influence the transferred charge and energy density in the discharge. From a chemical point of view high temperatures in the process gas have to be avoided because at elevated temperatures the equilibrium between atomic plus molecular oxygen and ozone is shifted to oxygen. Apart from this other parameters like process gas humidity, its purity, surface resistance of the dielectric influence the ozone yield. High ozone production rates (and concentrations) can be achieved by high energy densities as long as the cooling facility is sufficient efficient.

Comparing VD with SD ozone generators it is probable that SD devices will not exceed the efficiency of well optimized VD generators. The field strength distribution in SD leads in general to an average field strength which is farther from the optimal value than that in VD. Unexpected low ozone yields from SD generators fed with air may follow from enlarged concentrations of low nitrogen oxides.

\section{ACKNOWLEDGEMENT}

The authors are grateful for the financial support of the German Research Association (Deutsche Forschungsgemeinschaft, DFG).

\section{REFERENCES}

1. G. J. Pietsch and V. I. Gibalov, Proc. I2th Int. Conf. on Gas Discharges and Their Applications, vol. 2, 750-757, Greifswald, Germany (1997).

2. V. I. Gibalov, Russ. J. of Physical Chemistry 68, 1029-1033 (1994).

3. D. Braun, U. Küchler and G. Pietsch, Pure \& Appl. Chem. 60, 741-746 (1988). 